\title{
Homology rigidity of Grassmannians
}

Dedicated to Professor Wenjun Wu on his 90th birthday

Fang Li

Department of Mathematics, Jilin University

Haibao Duan*

Institute of Mathematics, Chinese Academy of Sciences

\begin{abstract}
Applying the theory of Gröbner basis to the Schubert presentation for the cohomology of Grassmannians $\left[\mathrm{DZ}_{1}\right]$, we extend the homology rigidity results known for the classical Grassmanians to the exceptional cases.
\end{abstract}

2000 Mathematics subject classification: 55 S37

Key words and phrases: Grassmannians; cohomology;

\section{Introduction}

Let $G$ be a compact connected Lie group with Lie algebra $L(G)$, exponential map exp : $L(G) \rightarrow G$, a maximal torus $T$, and a set $\Omega=\left\{\omega_{1}, \cdots, \omega_{n}\right\} \subset L(T)$ of fundamental dominant weights of $G$ (cf. [Hu]). For a weight $\omega \in \Omega$ let $H$ be the centralizer of the 1 -parameter subgroup $\{\exp (t \omega) \in G \mid t \in \mathbb{R}\}$. The homogeneous $G / H$ is a flag variety, called the Grassmannian of $G$ corresponding to $\omega$.

If $G$ is simple with rank $n$, we assume that the set $\Omega=\left\{\omega_{1}, \cdots, \omega_{n}\right\}$ is so ordered as the root-vertices in the Dynkin diagram of $G$ pictured in $[\mathrm{Hu}$, p.58]. With this convention we specify, in the table below, eight Grassmanianns $X=G / H$ associated to the exceptional Lie groups.

\begin{tabular}{|c||c|c|c|c|c|c|c|c|}
\hline \hline$G$ & $F_{4}$ & $F_{4}$ & $E_{6}$ & $E_{6}$ & $E_{7}$ & $E_{7}$ & $E_{7}$ & $E_{8}$ \\
\hline$\omega$ & $\omega_{1}$ & $\omega_{4}$ & $\omega_{2}$ & $\omega_{6}$ & $\omega_{1}$ & $\omega_{7}$ & $\omega_{2}$ & $\omega_{8}$ \\
$H$ & $C_{3} \cdot S^{1}$ & $B_{3} \cdot S^{1}$ & $A_{6} \cdot S^{1}$ & $D_{5} \cdot S^{1}$ & $D_{6} \cdot S^{1}$ & $E_{6} \cdot S^{1}$ & $A_{7} \cdot S^{1}$ & $E_{7} \cdot S^{1}$ \\
\hline
\end{tabular}

For a topological space $X$ let $[X, X]$ be the set of homotopy classes of selfmaps of $X$, and let $\operatorname{End}\left(H^{*}(X)\right)$ be the set of endomorphisms of the integral cohomology ring $H^{*}(X)$. It is known since 1970's that if $X$ is a flag variety, the correspondence

$$
[X, X] \rightarrow \operatorname{End}\left(H^{*}(X)\right)
$$

${ }^{*}$ Supported by NSFC 
assigning a map $f$ with its induced endomorphism $f^{*}$ is "nearly faithful" in the sense that it has finite kernel and finite cokernel with respect to the obvious monoid structure on both sets $(\mathrm{cf} .[\mathrm{GH}])$. Therefore, the problem of finding $\operatorname{End}\left(H^{*}(X)\right)$ for a flag manifold $X$ is a crucial step toward understanding all self-maps of $X$.

In this paper, we determine $\operatorname{End}\left(H^{*}(X)\right)$ for the eight Grassmannians tabulated above. Before stating our result, it is worthwhile to mention two earlier results on this topic.

It is known that, if $X$ is a Grassmannian, then

(1.1) $H^{r}(X)$ is torsion free and vanishes for odd $r$;

(1.2) $H^{2}(X)=\mathbb{Z}$ is generated by the Kaehlerian class $\alpha_{X}$ of $X$.

We note that, in view of (1.2), any endomorphism of $H^{*}(X)$ must map $\alpha_{X}$ to a multiple of itself.

Let $A=\underset{r \geq 0}{\oplus} A^{2 r}$ be a graded ring which vanishes in odd degrees (as being indicated), and let $p$ be an integer. The element $\psi^{p} \in \operatorname{End}(A)$ defined by

$$
\psi^{p}(x)=p^{r} x, x \in A^{2 r}
$$

is called the Adams operator on $A$ with order $p$.

Theorem 1 (Hoffmann $[\mathbf{H}, \mathbf{1 9 8 4}])$. Let $X$ be the Grassmannian $G_{n, k}$ of $k$-dimensional linear subspaces in $\mathbb{C}^{n}$, and let $h \in \operatorname{End}\left(H^{*}(X)\right)$ with $h\left(\alpha_{X}\right)=$ $p \alpha_{X}, p \neq 0$. Then

i) if $n \neq 2 k, h=\psi^{p}$;

ii) if $n=2 k$, there is the additional possibility $h=\psi^{p} \circ \tau$, where $\tau$ is the involution on $G_{2 k, k}$ that sends a $k$-dimensional linear subspaces $L \subset C^{n}$ to its othogonal complement $L^{\perp}$.

Theorem 2 (Duan, [D, 2003]). Let $X$ be the Grassmannian $\mathbb{C} S_{n}$ of complex structures on $R^{2 n}$ and let $h \in \operatorname{End}\left(H^{*}(X)\right)$ with $h\left(\alpha_{X}\right)=p \alpha_{X}, p \neq 0$. Then $h=\psi^{p}$

Hoffmann conjectured that Theorem 1 holds for the case $p=0$. In comparison it was shown in [D] that Theorem 2 is not true for $p=0$.

Turning to the exceptional Grassmannians under consideration, the case $X=E_{6} / A_{6} \cdot S^{1}$ will be of particular interest. Fix a maximal torus $T$ in $E_{6}$. Then

a) the Dynkind diagram of $E_{6}$ has the symmetry $\varphi$ given by reflection in the edge joinning the $2^{\text {nd }}$ and $4^{\text {th }}$ vertices [Hu, p.58], hence determines an automorphism $\varphi^{*}$ on $H^{*}\left(E_{6} / T\right)$ by $[\mathrm{P}]$;

b) the induced ring map $p^{*}: H^{*}\left(E_{6} / A_{6} \cdot S^{1}\right) \rightarrow H^{*}\left(E_{6} / T\right)$ of the fibration $p: E_{6} / T \rightarrow E_{6} / A_{6} \cdot S^{1}$ (corresponding to the inclusion $\left.T \subset A_{6} \cdot S^{1}\right)$ is injective, hence identify $H^{*}\left(E_{6} / A_{6} \cdot S^{1}\right)$ as a subring of $H^{*}\left(E_{6} / T\right)$. 
The main result of this paper is

Theorem 3. Let $X$ be one of the Grassmanians specified in the Table, and let $h \in \operatorname{End}\left(H^{*}(X)\right)$ with $h\left(\alpha_{X}\right)=p \alpha_{X}$. Then

i) if $X \neq E_{6} / A_{6} \cdot S^{1}, h=\psi^{p}$;

ii) if $X=E_{6} / A_{6} \cdot S^{1}$, there is the additional possibility $h=\psi^{p} \circ \tau$, where $\tau$ is the restriction of $\varphi^{*} \in \operatorname{Aut}\left(H^{*}\left(E_{6} / T\right)\right)$ to the subring $H^{*}\left(E_{6} / A_{6} \cdot S^{1}\right)$.

By establishing Theorem 3, we wish to demonstrate how the theory of Gröbner basis is used in extending the stricking rigidity property to Grassmannianns associated to the exceptional Lie groups.

The authors would like to thank Xu-an Zhao for clearifying the additional case ii) in Theorem 3, which first occurs to us from the calulation in Case 3 of $\S 4$.

\section{The ring $H^{*}(X ; \mathbb{Q})$ for a Grassmannian $X$}

If $X$ is a Grassmannian, the inclusion $\mathbb{Z} \rightarrow \mathbb{Q}$ of coefficients induces an injective ring map $H^{*}(X) \rightarrow H^{*}(X ; \mathbb{Q})$ by $(2.1)$. Therefore, it suffices to establish the Theorem for cohomology with rational coefficients (instead of integers).

In the classical approaches to $\operatorname{End}\left(H^{*}(X ; \mathbb{Q})\right)$ for $X=G_{n, k}, \mathbb{C} S_{n}$ (i.e. Theorems 1 and 2), the authors based their calculation on the existing presentations of $H^{*}(X ; \mathbb{Q})$ in the form of a quotient of a free polynomial ring

(2.1) $H^{*}(X ; \mathbb{Q})=\mathbb{Q}\left[y_{1}, \cdots, y_{n}\right] /\left\langle g_{1}, \cdots, g_{m}\right\rangle$,

where $g_{1}, \cdots, g_{m} \in \mathbb{Q}\left[y_{1}, \cdots, y_{n}\right]$, and where $\left\langle g_{1}, \cdots, g_{m}\right\rangle$ is the ideal generated by $g_{1}, \cdots, g_{m}$. In $\left[\mathrm{DZ}_{1}\right]$, a unified method computing the rings $H^{*}(X)$ for all Grassmannians $X$ has been developed. In particular, for those Grassmannians $X$ concerned by the Theorem, presentations of $H^{*}(X ; \mathbb{Q})$ in the form of $(2.1)$ can be deduced directly from $\left[\mathrm{DZ}_{1}\right.$, Theorems $\left.1-7\right]$ and $\left[\mathrm{DZ}_{2} ;(2.9)\right]$. We present these results in $(2.2)-(2.9)$ below, where the subscripts for the generators $y_{i}$ 's and relations $g_{j}$ 's are adopted to indicate their degrees in the fashion

$$
\operatorname{deg} y_{i}=2 i, \operatorname{deg} g_{j}=2 j ;
$$

and where $y_{1}$ is in the place of the Kaehlerian class $\alpha_{X}$ on $X$ (see (1.2)).

$(2.2) H^{*}\left(F_{4} / C_{3} \cdot S^{1} ; \mathbb{Q}\right)=\mathbb{Q}\left[y_{1}, y_{4}\right] /\left\langle g_{8}, g_{12}\right\rangle$, where

$g_{8}=24 y_{4}^{2}+y_{1}^{8}-12 y_{1}^{4} y_{4}$

$g_{12}=y_{1}^{12}-24 y_{1}^{8} y_{4}+144 y_{1}^{4} y_{4}^{2}-64 y_{4}^{3}$.

(2.3) $H^{*}\left(F_{4} / B_{3} \cdot S^{1} ; \mathbb{Q}\right)=\mathbb{Q}\left[y_{1}, y_{4}\right] /\left\langle g_{8}, g_{12}\right\rangle$, where

$g_{8}=3 y_{4}^{2}-y_{1}^{8}$

$g_{12}=26 y_{4}^{3}-5 y_{1}^{12}$.

(2.4) $H^{*}\left(E_{6} / A_{6} \cdot S^{1} ; \mathbb{Q}\right)=\mathbb{Q}\left[y_{1}, y_{3}, y_{4}\right] /\left\langle g_{8}, g_{9}, g_{12}\right\rangle$, where

$g_{8}=6 y_{4}^{2}-12 y_{1} y_{3} y_{4}+9 y_{1}^{2} y_{3}^{2}+3 y_{1}^{4} y_{4}-6 y_{1}^{5} y_{3}+y_{1}^{8}$;

$g_{9}=-2 y_{3}^{3}+6 y_{3} y_{1}^{2} y_{4}-3 y_{1}^{3} y_{3}^{2}+4 y_{3} y_{1}^{6}-3 y_{1}^{5} y_{4}-y_{1}^{9}$;

$g_{12}=4 y_{4}^{3}-y_{3}^{4}+6 y_{3}^{2} y_{1}^{2} y_{4}-4 y_{3}^{3} y_{1}^{3}-2 y_{3}^{2} y_{1}^{6}-9 y_{1}^{4} y_{4}^{2}+12 y_{1}^{5} y_{4} y_{3}$

$-6 y_{1}^{8} y_{4}+4 y_{1}^{9} y_{3}-y_{1}^{12}$. 
(2.5) $H^{*}\left(E_{6} / D_{5} \cdot S^{1} ; \mathbb{Q}\right)=\mathbb{Q}\left[y_{1}, y_{4}\right] /\left\langle g_{9}, g_{12}\right\rangle$, where

$g_{9}=2 y_{1}^{9}+3 y_{1} y_{4}^{2}-6 y_{1}^{5} y_{4}$

$g_{12}=y_{4}^{3}-6 y_{1}^{4} y_{4}^{2}+y_{1}^{12}$.

(2.6) $H^{*}\left(E_{7} / E_{6} \cdot S^{1} ; \mathbb{Q}\right)=\mathbb{Q}\left[y_{1}, y_{5}, y_{9}\right] /\left\langle g_{10}, g_{14}, g_{18}\right\rangle$, where

$g_{10}=y_{5}^{2}-2 y_{1} y_{9}$

$g_{14}=2 y_{5} y_{9}-9 y_{1}^{4} y_{5}^{2}+6 y_{1}^{9} y_{5}-y_{1}^{14}$

$g_{18}=y_{9}^{2}+10 y_{1}^{3} y_{5}^{3}-9 y_{1}^{8} y_{5}^{2}+2 y_{1}^{13} y_{5}$.

(2.7) $H^{*}\left(E_{7} / D_{6} \cdot S^{1} ; \mathbb{Q}\right)=\mathbb{Q}\left[y_{1}, y_{4}, y_{6}\right] /\left\langle g_{12}, g_{14}, g_{18}\right\rangle$, where

$g_{12}=3 y_{6}^{2}-y_{4}^{3}-3 y_{1}^{4} y_{4}^{2}-2 y_{1}^{6} y_{6}+2 y_{1}^{8} y_{4} ;$

$g_{14}=3 y_{4}^{2} y_{6}+3 y_{1}^{2} y_{6}^{2}+6 y_{1}^{2} y_{4}^{3}+6 y_{1}^{4} y_{4} y_{6}-3 y_{1}^{6} y_{4}^{2}-4 y_{1}^{8} y_{6}-2 y_{1}^{10} y_{4}+y_{1}^{14}$;

$g_{18}=45 y_{4}^{4} y_{1}^{2}+120 y_{4}^{2} y_{1}^{4} y_{6}+60 y_{4}^{3} y_{1}^{6}-52 y_{4}^{2} y_{1}^{10}-16 y_{1}^{6} y_{6}^{2}+80 y_{1}^{8} y_{6} y_{4}$

$-96 y_{1}^{12} y_{6}-48 y_{1}^{14} y_{4}+28 y_{1}^{18}+116 y_{6}^{3}+180 y_{1}^{2} y_{4} y_{6}^{2}$.

(2.8) $H^{*}\left(E_{8} / E_{7} \cdot S^{1} ; \mathbb{Q}\right)=\mathbb{Q}\left[y_{1}, y_{6}, y_{10}\right] /\left\langle g_{20}, g_{24}, g_{30}\right\rangle$, where

$g_{20}=3 y_{10}^{2}+10 y_{1}^{2} y_{6}^{3}+18 y_{1}^{4} y_{6} y_{10}-12 y_{1}^{10} y_{10}-18 y_{1}^{8} y_{6}^{2}+9 y_{1}^{14} y_{6}-y_{1}^{20} ;$

$g_{24}=5 y_{6}^{4}+30 y_{1}^{2} y_{6}^{2} y_{10}+15 y_{1}^{4} y_{10}^{2}-15 y_{1}^{14} y_{10}-15 y_{1}^{12} y_{6}^{2}+10 y_{1}^{18} y_{6}-y_{1}^{24}$;

$g_{30}=12 y_{1}^{4} y_{6} y_{10}^{2}+24 y_{1}^{14} y_{6} y_{10}+56 y_{1}^{24} y_{6}-36 y_{1}^{10} y_{10}^{2}-32 y_{10}^{3}+4 y_{6}^{5}-9 y_{1}^{30}$

$-48 y_{1}^{20} y_{10}+60 y_{1}^{6} y_{6}^{4}-64 y_{1}^{18} y_{6}^{2}+96 y_{1}^{8} y_{10} y_{6}^{2}-44 y_{1}^{12} y_{6}^{3}$.

(2.9) $H^{*}\left(E_{7} / A_{7} \cdot S^{1}, \mathbb{Q}\right)=\mathbb{Q}\left[y_{1}, y_{3}, y_{4}, y_{5}, y_{7}\right] /\left\langle g_{8}, g_{10}, g_{12}, g_{14}, g_{18}\right\rangle$, where

$g_{8}=6 y_{4}^{2}-4 y_{3} y_{5}+4 y_{1} y_{7}-12 y_{1} y_{3} y_{4}+9 y_{1}^{2} y_{3}^{2}+2 y_{1}^{3} y_{5}+3 y_{1}^{4} y_{4}-6 y_{1}^{5} y_{3}+y_{1}^{8}$;

$g_{10}=y_{5}^{2}-2 y_{3} y_{7}+y_{1}^{3} y_{7}$

$g_{12}=-4 y_{4}^{3}-2 y_{3}^{2} y_{1}^{6}+9 y_{1}^{4} y_{4}^{2}+6 y_{1}^{8} y_{4}-4 y_{1}^{9} y_{3}-24 y_{1} y_{4} y_{7}-12 y_{1}^{5} y_{4} y_{3}+8 y_{5} y_{7}$

$+4 y_{3} y_{1} y_{4}^{2}-18 y_{3}^{2} y_{1}^{2} y_{4}+12 y_{3} y_{1}^{2} y_{7}-3 y_{3}^{4}+y_{1}^{12}+16 y_{3}^{3} y_{1}^{3}$;

$g_{14}=y_{7}^{2}+3 y_{4} y_{5}^{2}+y_{5} y_{3}^{3}+3 y_{5} y_{3} y_{1}^{2} y_{4}-3 y_{5} y_{1}^{3} y_{3}^{2}+y_{5} y_{3} y_{1}^{6}-y_{5} y_{1} y_{4}^{2}-3 y_{5} y_{1}^{2} y_{7} ;$

$g_{18}=-8 y_{4}^{3} y_{5} y_{1}-4 y_{5} y_{7} y_{3}^{2}-2 y_{3} y_{1}^{7} y_{4}^{2}+y_{3}^{6}-6 y_{3} y_{1}^{8} y_{7}+18 y_{1}^{5} y_{3}^{2} y_{7}+15 y_{3}^{2} y_{1}^{4} y_{4}^{2}$

$-6 y_{3} y_{1}^{3} y_{4}^{3}+8 y_{4} y_{5} y_{3} y_{1}^{6}-12 y_{1} y_{5}^{2} y_{7}+y_{1}^{2} y_{4}^{4}+12 y_{4}^{2} y_{5}^{2}+y_{3}^{2} y_{1}^{12}-6 y_{1}^{9} y_{3}^{3}$

$+11 y_{3}^{4} y_{1}^{6}+8 y_{4} y_{5} y_{3}^{3}+24 y_{4}^{2} y_{5} y_{3} y_{1}^{2}+8 y_{3} y_{5}^{3}-18 y_{3}^{3} y_{1}^{5} y_{4}-2 y_{3}^{3} y_{1} y_{4}^{2}$

$+4 y_{5} y_{7} y_{1}^{6}+6 y_{3}^{4} y_{1}^{2} y_{4}-6 y_{3}^{5} y_{1}^{3}+6 y_{1}^{3} y_{4}^{2} y_{7}-18 y_{3} y_{1}^{4} y_{4} y_{7}-6 y_{3}^{3} y_{1}^{2} y_{7}$

$+6 y_{3}^{2} y_{1}^{8} y_{4}-4 y_{4} y_{7}^{2}+9 y_{1}^{4} y_{7}^{2}-24 y_{4} y_{5} y_{1}^{3} y_{3}^{2}-8 y_{5} y_{7} y_{1}^{3} y_{3}-12 y_{4} y_{5} y_{1}^{2} y_{7}$.

Remark. In (2.2)-(2.9), every generator $y_{i}$ is in fact a Schubert class of degree $i$ on the corresponding $X\left[\mathrm{DZ}_{1}\right]$.

\section{The algorithm}

As one can see from $(2.2)-(2.9)$ that the rings $H^{*}(X ; \mathbb{Q})$ vary considerably with respect to the Grassmannians $X$. However, with their common feature (2.1) in mind we may create some notations useful in a unified approach to $\operatorname{End}\left(H^{*}(X ; \mathbb{Q})\right)$.

For a polynomial ring $\mathbb{Q}\left[y_{1}, \cdots, y_{n}\right]$ graded by $\operatorname{deg} y_{i}>0$, its subspace of the homogeneous elements of degree $r$ has a canonical basis 
(3.1) $B^{r}=\left\{y^{\alpha}=y_{1}^{b_{1}} \cdots, y_{n}^{b n} \mid \alpha=\left(b_{1}, \cdots, b_{n}\right) \in \mathbb{N}^{n}, \operatorname{deg} y^{\alpha}=r\right\}$,

called the monomial basis of $\mathbb{Q}\left[y_{1}, \cdots, y_{n}\right]$ in degree $r$, where $\mathbb{N}^{n}$ is the set of all $n$-tuples $\alpha=\left(b_{1}, \cdots, b_{n}\right)$ of non-negative integers, considered as an ordered set with respect to the lexicographical order on $\mathbb{N}^{n}$.

For a subset $\left\{g_{1}, \cdots, g_{m}\right\} \subset \mathbb{Q}\left[y_{1}, \cdots, y_{n}\right]$ let $\mathcal{G}$ be the Gröbner basis of the ideal $\left\langle g_{1}, \cdots, g_{m}\right\rangle$ [E]. The package "Gbasis" in MAPLE [E] has the function

In: A subset $\left\{g_{1}, \cdots, g_{m}\right\} \subset \mathbb{Q}\left[y_{1}, \cdots, y_{n}\right]$;

Out: the Gröbner basis $\mathcal{G}$ of $\left\langle g_{1}, \cdots, g_{m}\right\rangle$,

whose importance is shown in the next result.

Lemma 1. Each polynomial $f \in \mathbb{Q}\left[y_{1}, \cdots, y_{n}\right]$ determines a unique element $h \in \mathbb{Q}\left[y_{1}, \cdots, y_{n}\right]$, called the residue of $f$ module $\left\langle g_{1}, \cdots, g_{m}\right\rangle$, that satisfies

i) $f \equiv h \bmod \mathcal{G}$;

ii) $f \in\left\langle g_{1}, \cdots, g_{m}\right\rangle$ if and only if $h=0$.

Moreover, the package "Normal" in MAPLE [E] has the function to implement $h$ from $f$.

We may now clarify the algorithm by which Theorem 3 is established. An endomorphism $f$ of the ring (2.1) can be regarded as an endomorphism of the free polynomial ring $\mathbb{Q}\left[y_{1}, \cdots, y_{n}\right]$ which preserves the ideal $\left\langle g_{1}, \cdots, g_{m}\right\rangle$. Based on this observation we can assume that $f$ is given by

$$
f\left(y_{i}\right)=\sum_{y^{\alpha} \in B^{s_{i}}} c_{\alpha}^{i} \cdot y^{\alpha}, s_{i}=\operatorname{deg} y_{i}, c_{\alpha}^{i} \in \mathbb{Q}, i=1, \cdots, n
$$

that are subject to the restrictions

$$
f\left(g_{j}\left(y_{1}, \cdots, y_{n}\right)\right)=g_{j}\left(f\left(y_{1}\right), \cdots, f\left(y_{n}\right)\right) \in\left\langle g_{1}, \cdots, g_{m}\right\rangle, \quad j=1, \cdots, m
$$

where the equality in (3.3) comes from the fact that $f$ is a ring map. Assume that the residue of $g_{j}\left(f\left(y_{1}\right), \cdots, f\left(y_{n}\right)\right)$ module $\left\langle g_{1}, \cdots, g_{m}\right\rangle$ is

(3.4) $h_{j}=\sum_{y^{\alpha} \in B^{t_{j}}} b_{\alpha}^{j} \cdot y^{\alpha}, t_{j}=\operatorname{deg} g_{j}$,

where $b_{\alpha}^{j}$ are certain polynomials in the $c_{\alpha}^{i}$ 's in (3.2). Then (3.3) is equivalent to the system

$(3.5) \mathcal{S}:\left\{b_{\alpha}^{j}=0 \mid 1 \leq j \leq m, y^{\alpha} \in B^{t_{j}}\right\}$

by the Lemma. In other word, (3.5) constitutes all the constraints that the $c_{\alpha}^{i}$ 's in (3.2) must satisfy. Finally, we remark that the package "Solve" or "Gsolve" in MAPLE [E] has the function to produce all the solutions to the system (3.5). 


\section{Proof of the Theorem}

Case 1. $X=F_{4} / C_{3} \cdot S^{1}$ (see (2.2)). In accordance with the order $y_{1}>y_{4}$, the Gröbner basis of the ideal $\left\langle g_{8}, g_{12}\right\rangle$ is:

$$
\mathcal{G}=\left\{24 y_{4}^{2}+y_{1}^{8}-12 y_{1}^{4} y_{4}, 3 y_{1}^{4} y_{4}^{2}-28 y_{4}^{3}, y_{4}^{4}\right\}
$$

Assume that $f \in \operatorname{End}\left(H^{*}(X ; \mathbb{Q})\right)$ is given by

$$
f\left(y_{1}\right)=k y_{1}, \quad f\left(y_{4}\right)=a y_{1}^{4}+b y_{4}, \quad k, a, b \in \mathbb{Q} .
$$

Then the residues of $f\left(g_{i}\right), i=8,12$, module $\left\langle g_{8}, g_{12}\right\rangle$ are (Lemma 1)

$$
\begin{aligned}
h_{8}= & 12\left(24 a^{2}+k^{8}-12 k^{4} a+4 b a-k^{4} b\right) y_{1}^{4} y_{4} \\
& +12\left(-48 a^{2}+24 k^{4} a-2 k^{8}+2 b^{2}\right) y_{4}^{2} \\
h_{12}= & -\left(-1344 k^{4} b^{2}+1792 b^{2} a-832 k^{12}+53248 a^{3}-25344 k^{4} b a\right. \\
& \left.+64 b^{3}-119808 k^{4} a^{2}+19968 k^{8} a+2112 k^{8} b+16896 b a^{2}\right) y_{4}^{3} .
\end{aligned}
$$

Applying Solve to the corresponding system (3.5) yields that

$$
a=0, b=k^{4}, k=k .
$$

Case 2. $X=F_{4} / B_{3} \cdot S^{1}$ (see (2.3)). In accordance with the order $y_{1}>y_{4}$, the Gröbner basis of the ideal $\left\langle g_{8}, g_{12}\right\rangle$ is:

$$
\mathcal{G}=\left\{y_{1}^{8}-3 y_{4}^{2}, 15 y_{4}^{2} y_{1}^{4}-26 y_{4}^{3}, y_{4}^{4}\right\} .
$$

Assume that $f \in \operatorname{End}\left(H^{*}(X ; \mathbb{Q})\right)$ is given by

$$
f\left(y_{1}\right)=k y_{1}, \quad f\left(y_{4}\right)=a y_{1}^{4}+b y_{4}, \quad k, a, b \in \mathbb{Q} .
$$

Then the residues of $f\left(g_{i}\right), i=8,12$, module $\left\langle g_{8}, g_{12}\right\rangle$ are (Lemma 1)

$$
\begin{aligned}
& h_{8}=3\left(3 a^{2}-k^{8}+b^{2}\right) y_{4}^{2}+6 a b y_{1}^{4} y_{4} ; \\
& h_{12}=\frac{1}{5}\left(676 a^{3}-130 k^{12}+676 a b^{2}+1170 a^{2} b+130 b^{3}\right) y_{4}^{3}
\end{aligned}
$$

Applying Solve to the corresponding system (3.5) yields that

$$
a=0, b=k^{4}, k=k .
$$

Case 3. $X=E_{6} / A_{6} \cdot S^{1}$ (see (2.4)). In accordance with the order $y_{1}>y_{3}>y_{4}$, the Gröbner basis of the ideal $\left\langle g_{8}, g_{9}, g_{12}\right\rangle$ is

$$
\begin{aligned}
\mathcal{G}= & \left\{6 y_{4}^{2}-12 y_{1} y_{3} y_{4}+9 y_{1}^{2} y_{3}^{2}+3 y_{1}^{4} y_{4}-6 y_{1}^{5} y_{3} ;\right. \\
& y_{3} y_{1}^{6}-3 y_{1}^{3} y_{3}^{2}+3 y_{3} y_{1}^{2} y_{4}+y_{3}^{3}-3 y_{4}^{2} y_{1} \\
& 3 y_{3}^{2} y_{1}^{5}-8 y_{3}^{3} y_{1}^{2}-3 y_{4}^{2} y_{1}^{3}+12 y_{4} y_{3}^{2} y_{1}-6 y_{4}^{2} y_{3} \\
& \left.2 y_{3}^{4}-30 y_{3}^{2} y_{1}^{2} y_{4}+33 y_{4}^{2} y_{3} y_{1}-y_{3}^{3} y_{1}^{3}-22 y_{4}^{3}+12 y_{1}^{5} y_{4} y_{3} ; \cdots\right\},
\end{aligned}
$$

where we have omitted the elements in $\mathcal{G}$ with degree $>\max \left\{\operatorname{deg} g_{i}\right\}$ since they play no further role in the process. Assume that $f \in \operatorname{End}\left(H^{*}(X ; \mathbb{Q})\right)$ is given by 


$$
f\left(y_{1}\right)=k y_{1}, \quad f\left(y_{3}\right)=a_{1} y_{1}^{3}+a_{2} y_{3}, \quad f\left(y_{4}\right)=b_{1} y_{1}^{4}+b_{2} y_{1} y_{3}+b_{3} y_{4},
$$

where $k, a_{1}, a_{2}, b_{1}, b_{2}, b_{3} \in \mathbb{Q}$. Then the residues of $f\left(g_{i}\right), i=8,9$, module $\left\langle g_{8}, g_{9}, g_{12}\right\rangle$ are (Lemma 1)

$$
\begin{aligned}
& h_{8}=3\left(4 b_{2} b_{3}+24 b_{1}^{2}+36 k^{2} a_{1}^{2}-48 k a_{1} b_{1}+12 k^{4} b_{1}-24 k^{5} a_{1}+4 k^{8}\right. \\
& \left.-4 k a_{2} b_{3}\right) y_{1} y_{3} y_{4}+3\left(12 b_{1}^{2}+2 k^{8}-24 k a_{1} b_{1}+18 k^{2} a_{1}^{2}+6 k^{4} b_{1}\right. \\
& \left.-12 k^{5} a_{1}+4 b_{1} b_{2}+k^{4} b_{2}-2 k^{5} a_{2}-4 k a_{1} b_{2}-4 k a_{2} b_{1}+6 k^{2} a_{1} a_{2}\right) y_{1}^{5} y_{3} \\
& +3\left(3 k^{2} a_{2}^{2}-18 b_{1}^{2}-4 k a_{2} b_{2}-9 k^{4} b_{1}-27 k^{2} a_{1}^{2}+2 b_{2}^{2}+36 k a_{1} b_{1}-3 k^{8}\right. \\
& \left.+18 k^{5} a_{1}\right) y_{1}^{2} y_{3}^{2}+3\left(12 k^{5} a_{1}-2 k^{8}-6 k^{4} b_{1}+2 b_{3}^{2}-12 b_{1}^{2}+24 k a_{1} b_{1}\right. \\
& \left.-18 k^{2} a_{1}^{2}\right) y_{4}^{2}+3\left(k^{4} b_{3}-k^{8}+6 k^{5} a_{1}-3 k^{4} b_{1}-4 k a_{1} b_{3}-6 b_{1}^{2}+4 b_{1} b_{3}\right. \\
& \left.+12 k a_{1} b_{1}-9 k^{2} a_{1}^{2}\right) y_{1}^{4} y_{4} \\
& h_{9}=-18 a_{1}^{2} a_{2}+12 k^{6} a_{2}+72 k^{2} a_{1} b_{1}-24 a_{1}^{3}-12 k^{9}-36 k^{3} a_{1}^{2}+48 k^{6} a_{1} \\
& \left.-36 k^{5} b_{1}+18 k^{2} a_{1} b_{2}+18 k^{2} a_{2} b_{1}-18 k^{3} a_{1} a_{2}-9 k^{5} b_{2}\right) y_{4}^{2} y_{1} \\
& +\left(36 k^{6} a_{1}-27 k^{5} b_{1}-9 k^{9}-27 k^{3} a_{1}^{2}-18 a_{1}^{2} a_{2}+12 k^{6} a_{2}-9 k^{5} b_{2}\right. \\
& -6 a_{1} a_{2}^{2}+18 k^{2} a_{1} b_{2}+18 k^{2} a_{2} b_{1}-18 k^{3} a_{1} a_{2}+54 k^{2} a_{1} b_{1}-18 a_{1}^{3} \\
& \left.+6 k^{2} a_{2} b_{2}-3 k^{3} a_{2}^{2}\right) y_{1}^{3} y_{3}^{2}+\left(9 k^{5} b_{1}+6 a_{1}^{3}+3 k^{9}+9 k^{3} a_{1}^{2}-12 k^{6} a_{1}\right. \\
& \left.-18 k^{2} a_{1} b_{1}+6 k^{2} a_{1} b_{3}-3 k^{5} b_{3}\right) y_{1}^{5} y_{4}+\left(-24 k^{6} a_{1}+18 k^{5} b_{1}+6 a_{1}^{2} a_{2}\right. \\
& -4 k^{6} a_{2}+3 k^{5} b_{2}+6 k^{9}-36 k^{2} a_{1} b_{1}-6 k^{2} a_{1} b_{2}-6 k^{2} a_{2} b_{1}+6 k^{3} a_{1} a_{2} \\
& \left.+12 a_{1}^{3}+18 k^{3} a_{1}^{2}-2 a_{2}^{3}\right) y_{3}^{3}+\left(6 k^{9}+12 a_{1}^{3}+18 a_{1}^{2} a_{2}-18 k^{2} a_{1} b_{2}\right. \\
& -18 k^{2} a_{2} b_{1}+18 k^{3} a_{1} a_{2}-12 k^{6} a_{2}+9 k^{5} b_{2}+18 k^{3} a_{1}^{2}-24 k^{6} a_{1} \\
& \left.+18 k^{5} b_{1}-36 k^{2} a_{1} b_{1}+6 k^{2} a_{2} b_{3}\right) y_{3} y_{1}^{2} y_{4} \text {. }
\end{aligned}
$$

With $k, a_{1}, a_{2}, b_{1}, b_{2}, b_{3} \in \mathbb{Q}$ the corresponding system (3.5) has two solutions

$$
\begin{aligned}
& \left\{a_{1}=k^{3}, a_{2}=-k^{3}, b_{1}=k^{4}, b_{2}=-2 k^{4}, b_{3}=k^{4}\right\}, \\
& \left\{a_{1}=0, a_{2}=k^{3}, b_{1}=0, b_{2}=0, b_{3}=k^{4}\right\},
\end{aligned}
$$

and with respect to the first solution, the residue $h_{12}$ of $f\left(g_{12}\right)$ is trivial. This establish the Theorem for the current situation.

Case 4. $X=E_{6} / D_{5} \cdot S^{1}$ (see (2.5)). In accordance with the order $y_{1}>y_{4}$, the Gröbner basis of the ideal $\left\langle g_{9}, g_{12}\right\rangle$ is:

$$
\begin{aligned}
\mathcal{G}= & \left\{2 y_{1}^{9}+3 y_{1} y_{4}^{2}-6 y_{1}^{5} y_{4}, 6 y_{4} y_{1}^{8}-15 y_{1}^{4} y_{4}^{2}+2 y_{4}^{3}, 3 y_{4}^{2} y_{1}^{5}-7 y_{4}^{3} y_{1},\right. \\
& \left.y_{4}^{3} y_{1}^{4}-2 y_{4}^{4}, y_{4}^{4} y_{1}, y_{4}^{5}\right\} .
\end{aligned}
$$

Assume that $f \in \operatorname{End}\left(H^{*}(X ; \mathbb{Q})\right)$ is given by

$$
f\left(y_{1}\right)=k y_{1}, \quad f\left(y_{4}\right)=a y_{1}^{4}+b y_{4}, \quad k, a, b \in \mathbb{Q} .
$$

Then the residues of $f\left(g_{i}\right), i=9,12$, module $\left\langle g_{9}, g_{12}\right\rangle$ are (Lemma 1)

$$
\begin{aligned}
h_{9}= & \frac{3}{2}\left(-2 k^{8}+6 k^{4} a-3 a^{2}+2 b^{2}\right) k y_{1} y_{4}^{2}+\frac{3}{2}\left(4 k^{8}-12 k^{4} a+6 a^{2}\right. \\
& \left.-4 k^{4} b+4 a b\right) k y_{1}^{5} y_{4} ; \\
h_{12}= & \frac{1}{2}\left(-2 a^{3}-2 k^{12}+12 k^{4} a^{2}-2 a^{2} b+2 b^{3}+8 k^{4} a b\right) y_{4}^{3}+\frac{1}{2}\left(12 a^{3}\right. \\
& \left.+12 k^{12}-60 k^{4} a b+6 a b^{2}-12 k^{4} b^{2}-72 k^{4} a^{2}+15 a^{2} b\right) y_{1}^{4} y_{4}^{2} .
\end{aligned}
$$


Applying Solve to the corresponding system (3.5) yields that

$$
a=0, b=k^{4}, k=k .
$$

Case 5. $X=E_{7} / E_{6} \cdot S^{1}$ (see (2.6)). In accordance with the order $y_{1}>y_{4}>y_{9}$, the Gröbner basis of the ideal $\left\langle g_{10}, g_{14}, g_{18}\right\rangle$ is:

$$
\begin{aligned}
\mathcal{G}= & \left\{-2 y_{5} y_{9}+9 y_{1}^{4} y_{5}^{2}-6 y_{1}^{9} y_{5}+y_{1}^{14}, y_{9}^{2}+10 y_{1}^{3} y_{5}^{3}-9 y_{1}^{8} y_{5}^{2}+2 y_{1}^{13} y_{5},\right. \\
& \left.-y_{5}^{2}+2 y_{1} y_{9}, \cdots\right\},
\end{aligned}
$$

where the elements in $\mathcal{G}$ with degree $>\max \left\{\operatorname{deg} g_{i}\right\}$ has been omitted because they play no role in the latter course. Assume that $f \in \operatorname{End}\left(H^{*}(X ; \mathbb{Q})\right)$ is given by

$$
f\left(y_{1}\right)=k y_{1}, f\left(y_{5}\right)=a_{1} y_{1}^{5}+a_{2} y_{5}, f\left(y_{9}\right)=b_{1} y_{1}^{9}+b_{2} y_{1}^{4} y_{5}+b_{3} y_{9},
$$

where $k, a_{1}, a_{2}, b_{1}, b_{2}, b_{3} \in \mathbb{Q}$. Then the residues of $f\left(g_{i}\right), i=10,14,18$, module $\left\langle g_{10}, g_{14}, g_{18}\right\rangle$ are (Lemma 1)

$$
\begin{aligned}
h_{10}= & \left(2 a_{2}^{2}-2 k b_{3}\right) y_{1} y_{9}+\left(2 a_{1} a_{2}-2 k b_{2}\right) y_{1}^{5} y_{5}+\left(a_{1}^{2}-2 k b_{1}\right) y_{1}^{10} ; \\
h_{14}= & 2\left(81 k^{4} a_{1}^{2}+2 a_{2} b_{2}-9 k^{4} a_{2}^{2}-18 a_{1} b_{1}-54 k^{9} a_{1}+9 k^{14}+a_{1} b_{3}\right) y_{9} y_{1}^{5} \\
& +2\left(a_{1} b_{2}+a_{2} b_{1}+3 k^{9} a_{2}+6 a_{1} b_{1}-27 k^{4} a_{1}^{2}+18 k^{9} a_{1}-9 k^{4} a_{1} a_{2}\right. \\
& \left.-3 k^{14}\right) y_{1}^{9} y_{5}+2\left(a_{2} b_{3}-k^{14}+2 a_{1} b_{1}-9 k^{4} a_{1}^{2}+6 k^{9} a_{1}\right) y_{5} y_{9} ; \\
h_{18}= & \left(2 b_{1} b_{3}+270 k^{3} a_{1}^{2} a_{2}-162 k^{8} a_{1} a_{2}+72 k^{13} a_{1}+18 b_{1} b_{2}+18 k^{13} a_{2}\right. \\
& \left.-18 k^{8} a_{2}^{2}+360 k^{3} a_{1}^{3}-324 k^{8} a_{1}^{2}+60 k^{3} a_{1} a_{2}^{2}+2 b_{2}^{2}+36 b_{1}^{2}\right) y_{9} y_{1}^{9} \\
& +\left(522 k^{8} a_{1}^{2}-580 k^{3} a_{1}^{3}+2 b_{2} b_{3}-300 k^{3} a_{1}^{2} a_{2}-58 b_{1}^{2}-116 k^{13} a_{1}\right. \\
& \left.+180 k^{8} a_{1} a_{2}-20 b_{1} b_{2}-20 k^{13} a_{2}+20 k^{3} a_{2}^{3}\right) y_{9} y_{5} y_{1}^{4}+\left(b_{3}^{2}-15 k^{3} a_{1}^{2} a_{2}\right. \\
& \left.+9 k^{8} a_{1} a_{2}-3 b_{1}^{2}-k^{13} a_{2}-30 k^{3} a_{1}^{3}+27 k^{8} a_{1}^{2}-6 k^{13} a_{1}-b_{1} b_{2}\right) y_{9}^{2} .
\end{aligned}
$$

Applying Solve to the corresponding system (3.5) yields that

$$
a_{1}=b_{1}=b_{2}=0, a_{2}=k^{5}, b=k^{9}, k=k .
$$

Case 6. $X=E_{7} / D_{6} \cdot S^{1}, E_{8} / E_{7} \cdot S^{1}$ or $E_{7} / A_{7} \cdot S^{1}$. Procedure the same as the above establish Theorem 3 for these cases. Only the expressions of the corresponding $h_{i}$ 's are very lengthy, and the package "Gsolve" in MAPLE appears more effective than the "Solve" when implementing the corresponding system (3.5). We omit the details and refer to the thesis [L] of the first author for thorough discussions on these cases.

\section{References}

[D] H. Duan, Self-maps of the Grassmannian of complex structures, Compositio Math., 132 (2002), 159-175.

[DZ $\left.{ }_{1}\right]$ H. Duan and Xuezhi Zhao, The Chow rings of generalized Grassmannians, arXiv: math.AG/0511332

$\left[\mathrm{DZ}_{2}\right]$ H. Duan and Xuezhi Zhao, The integral cohomology of complete flag manifolds, arXiv: math.AT/0801.2444. 
[E] K. Ernic, A Guide To Maple, Springer Verlag, 1999.

[GH] H. Glover and W. Homer, Self-maps of flag manifolds, Trans. AMS 267(1981), 423-434.

$\left[\mathrm{H}_{1}\right]$ M. Hoffman, Endomorphisms of the cohomology of complex Grassmannians, Trans, AMS 281 (1984), 745-740.

$\left[\mathrm{H}_{2}\right]$ M. Hoffman, On fixed point free maps of the complex flag manifold, Indiana Math. J., 33(1984), 249-255.

[Hu] J. E. Humphreys, Introduction to Lie algebras and representation theory, Graduated Texts in Math. 9, Springer-Verlag New York, 1972.

[L] F. Li, Endomorphisms of the cohomology ring of a generalized Grassmannian, Thesis, Jilin University.

[P] S. Papadima, Rigidity properties of compact Lie groups modulo maximal tori, Math. Ann. 275(1987), 637-652. 